\title{
New Proposed Method for Solving Assignment Problem and Comparative Study with the Existing Methods
}

\author{
Humayra Dil Afroz ${ }^{1}$, Dr. Mohammad Anwar Hossen ${ }^{2}$ \\ 1 DepartmentofMat hematics, ComillaUniversity ,Banglades $h$ \\ 2 Departmentof Mat hematics, ComillaUniversity ,Banglades $h$
}

\begin{abstract}
Assignment problem is an important problem in mathematics and is also discuss in real physical world. In this paper we attempt to introduce a new proposed approach for solving assignment problem with algorithm and solution steps. We examine a numerical example by using new method and compute by existing two methods. Also we compare the optimal solutions among this new method and two existing method .The proposed method isa systematic procedure, easy to apply for solving assignment problem.
\end{abstract}

Keywords: Assignment problem, Hungarian assignment method (HA-method), Matrix one's assignment method (MOA-method), Proposed method, Optimization.

\section{Introduction}

The assignment problem is one of the main problems while assigning task to the worker. It is one of the fundamental combinatorial optimization problems in the branch of optimization or operation research in Mathematics. It is one of the special cases of transportation problems. This is particularly important in the theory of decision making. This problem finds numerous applications in production planning, telecommunication, VLSI design, economics etc. In a normal case of assignment problem where the objective is to assign the available resources to the activity going on so as to get the minimum cost or maximum total benefits of allocation.

In this paper we developed a solution method for assignment problem. The corresponding method has been formulated and numerical example has been considered to illustrate the method. Finally we compare the optimal solutions among new method and two existing methods.

\section{Mathematical Formulation of Assignment Problem}

Each assignment problem has a matrix associated with it. Generally the row contains the objects or people we wish to assign, and the column comprise the jobs or tasks we want them assigned to. Consider a problem of assignment of $\mathrm{n}$ resources to $\mathrm{m}$ activities so as to minimize the overall cost or time in such a way that each resource can associate with one and only one job. The cost matrix $\left(C_{i j}\right)$ is given as under:

Table 1.1 Approach of Assignment Problem

\begin{tabular}{|c|c|c|c|c|c|c|}
\hline & & & $\mathrm{AC}$ & & & \\
\hline & & $A_{1}$ & $A_{2}$ & …... & $A_{n}$ & \\
\hline & $\mathbf{R}_{1}$ & $\mathrm{C}_{11}$ & $C_{12}$ & $\cdots \ldots$ & $C_{1 n}$ & 1 \\
\hline esource & $R_{2}$ & $C_{21}$ & $\mathrm{C}_{22}$ & ....... & $C_{2 n}$ & 1 \\
\hline & . & - & - & - & - & \\
\hline & . & - & - & - & - & . \\
\hline & . & - & - & - & . & . \\
\hline & $R_{n}$ & $\mathrm{C}_{\mathrm{n} 1}$ & $\mathrm{C}_{\mathrm{n} 2}$ & ....... & $\mathrm{C}_{\mathrm{nn}}$ & 1 \\
\hline Required & & 1 & 1 & ....... & 1 & \\
\hline
\end{tabular}

The cost matrix is same as that of a transportation problem except that availability at each of the resource and the requirement at each of the destinations is unity.

Let $x_{i j}$ denote the assignment of $i^{\text {th }}$ resource to $j^{\text {th }}$ activity, such that

$$
x_{i j}=\left\{\begin{array}{rr}
1 ; & \text { if resource } \mathrm{i} \text { is assigned to activity } \mathrm{j} \\
0 ; & \text { otherwise }
\end{array}\right.
$$

Then the mathematical formulation of the assignment problem is

Minimize $z=\sum_{\mathrm{i}=1}^{n} \sum_{j=1}^{n} \mathrm{c}_{\mathrm{ij}} \mathrm{x}_{\mathrm{ij}}$

Subject to the constraints

$\sum_{\mathrm{i}=1}^{\mathrm{n}} \mathrm{x}_{\mathrm{ij}}=1$ and $\sum_{\mathrm{j}=1}^{\mathrm{n}} \mathrm{x}_{\mathrm{ij}}=1: \mathrm{x}_{\mathrm{ij}}=0$ or 1

For all $\mathrm{i}=1,2, \ldots \ldots \mathrm{n}$ and $\mathrm{j}=1,2, \ldots \ldots \mathrm{n}$.

\section{New Approach For Solving Assignment Problem}

In this section we introduce a new approach for solving Assignment problem with the help of HA-method and MOA-method but different from them. This new method is easy procedure to solve Assignment problem. Also an example is solved by this method and the result is compared to HA-method and MOA-method.

Now we consider the assignment matrix where $c_{i j}$ is the cost of assigning ith job to jth machine. 


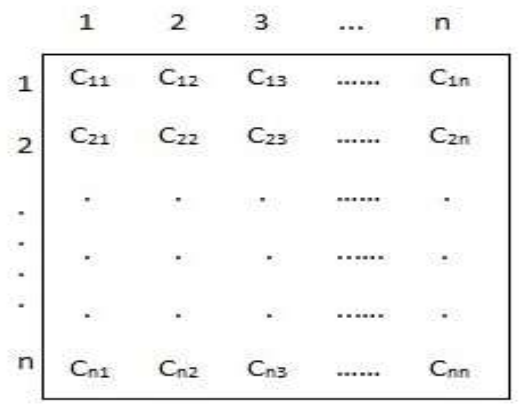

\section{Proposed Method: Subtract Row and Add One Assignment Method}

The proposed algorithm of proposed method is as follows:

Step 1: Find the smallest number (cost) of each row. Subtract this smallest number from every number in that row. Step 2: Now add 1 to all element and we get at least one ones in each row. Then make assignment in terms of ones. If there are some rows and columns without assignment, then we cannot get the optimum solution. Then we go to the next step.

Step 3: Draw the minimum number of lines passing through all ones by using the following procedure:

i. $\quad \operatorname{Mark}(\sqrt{ })$ rows that do not have assignments.

ii. Mark $(\sqrt{ })$ columns that have crossed ones in that marked rows.

iii. Mark $(\sqrt{ })$ rows that have assignments in marked columns.

iv. Repeat (b) and (c) till no more rows or columns can be marked.

v. Draw straight lines through all unmarked rows and marked columns.

If the number of lines drawn is equal to the number of rows or columns, then the current solution is optimal solution. Otherwise go to next step.

Step 4: Select the smallest number of the reduced matrix not covered by the lines. Divide all uncovered numbers by this smallest number. Other numbers covered by lines remain unchanged. Then we get some new ones in row and column. Again make assignment in terms of ones.

Step 5: If we cannot get the optimal assignment in each row and column. Then repeat steps (3) and (4) successively till an optimum solution is obtained.

\section{Numerical Comparison Of Existing Methods With Proposed Method}

1. Solve the following assignment problem using Proposed Method.

Consider the problem of assigning five jobs to five persons. The assignment costs are given below.

\begin{tabular}{|l|lllll|}
\hline \multirow{2}{*}{ Persons } & \multicolumn{5}{|c|}{ Jobs } \\
\hline A & 1 & 2 & 3 & 4 & 5 \\
B & 8 & 4 & 2 & 6 & 1 \\
C & 0 & 9 & 5 & 5 & 4 \\
D & 3 & 8 & 9 & 2 & 6 \\
E & 4 & 3 & 1 & 0 & 3 \\
\hline
\end{tabular}

Determine the optimum assignment schedule and minimum assignment cost.

Solution:

Step 1: Find the minimum element of each row and subtract it from each element in that row. Then the reduced matrix is as follows:

\begin{tabular}{l|lllll|}
\multicolumn{1}{c}{} & \multicolumn{1}{l}{1} & 2 & 3 & 4 & 5 \\
A & 7 & 3 & 1 & 5 & 0 \\
B & 0 & 9 & 5 & 5 & 4 \\
C & 1 & 6 & 7 & 0 & 4 \\
D & 4 & 3 & 1 & 0 & 3 \\
E & 4 & 0 & 3 & 4 & 0 \\
\cline { 2 - 5 } & & & & &
\end{tabular}

Step 2: Now add 1 to all element.

\begin{tabular}{l|lllll|} 
A & 1 & 2 & 3 & 4 & 5 \\
\hline B & 8 & 4 & 2 & 6 & 1 \\
C & 1 & 10 & 6 & 6 & 5 \\
D & 5 & 7 & 8 & 1 & 5 \\
& 5 & 4 & 2 & 1 & 4 \\
& 1 & 4 & 5 & 1 \\
\hline
\end{tabular}


Step 3: Now make initial assignment.

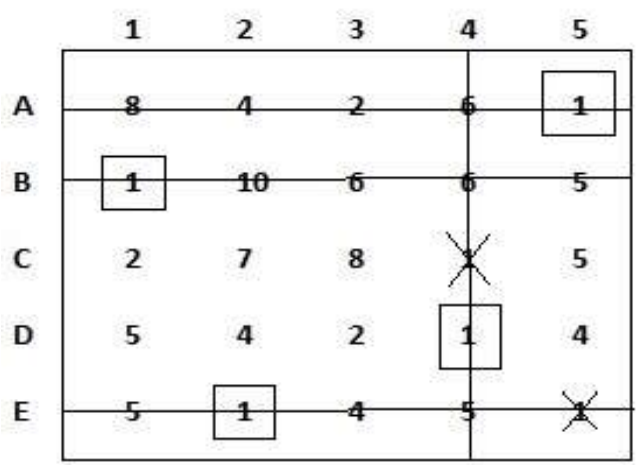

Here, 3rd row and 3rd column do not have any assignment. Thus the solution is not optimum and we go to next.

Step 4: Select the smallest number of the reduced matrix not covered by the lines. Divide all uncovered number by this smallest number. Other numbers covered by lines remain unchanged. Then make assignment again.

\begin{tabular}{|c|c|c|c|c|c|}
\hline & 1 & 2 & 3 & 4 & 5 \\
\hline A & 8 & 4 & 2 & 6 & 1 \\
\hline B & 1 & 10 & 6 & 6 & 5 \\
\hline C & $x$ & 3.5 & 4 & 1 & 2.5 \\
\hline D & 2.5 & 2 & 1 & & 2 \\
\hline$E$ & 5 & 1 & 4 & 5 & $x$ \\
\hline
\end{tabular}

Here we see that all ones are either assigned or crossed out. That is, the total assigned one's is 5 which is equal to the number of rows or columns. And the solution is $(1,5),(2,1),(3,4),(4,3),(5,2)$.

So the total cost $=1+0+2+1+5=09$.

\section{Solve the following assignment problem using Hungarian Assignment Method.}

Consider the problem of assigning five jobs to five persons. The assignment costs are given below.

\begin{tabular}{|l|lllll|}
\hline \multirow{2}{*}{ Persons } & \multicolumn{5}{|c|}{ Jobs } \\
& 1 & 2 & 3 & 4 & 5 \\
\hline A & 8 & 4 & 2 & 6 & 1 \\
B & 0 & 9 & 5 & 5 & 4 \\
D & 3 & 8 & 9 & 2 & 6 \\
E & 4 & 3 & 1 & 0 & 3 \\
\hline
\end{tabular}

Determine the optimum assignment schedule and minimum assignment cost.

Solution:

Step 1: Select the minimum element in each row and subtract this element from every element in that row.

\begin{tabular}{l|lllll|}
\multicolumn{1}{c}{} & \multicolumn{1}{c}{1} & 2 & 3 & 4 & 5 \\
A & 7 & 3 & 1 & 5 & 0 \\
B & 0 & 9 & 5 & 5 & 4 \\
C & 1 & 6 & 7 & 0 & 3 \\
D & 4 & 3 & 1 & 0 & 3 \\
E & 4 & 0 & 3 & 4 & 0 \\
\hline
\end{tabular}

Step 2: Select the minimum element in each column and subtract this element from every element in that column.

\begin{tabular}{l|lllll|}
\multicolumn{1}{c}{} & \multicolumn{1}{c}{1} & 2 & 3 & 4 & 5 \\
A & 7 & 3 & 0 & 5 & 0 \\
B & 0 & 9 & 4 & 5 & 4 \\
C & 1 & 6 & 6 & 0 & 3 \\
D & 4 & 3 & 0 & 0 & 3 \\
E & 4 & 0 & 2 & 4 & 0 \\
\hline
\end{tabular}


Step 3: Make initial assignment.

\begin{tabular}{|c|c|c|c|c|c|}
\hline & 1 & 2 & 3 & 4 & 5 \\
\hline A & 7 & 3 & $\not 2$ & 5 & 0 \\
\hline B & 0 & 9 & 4 & 5 & 4 \\
\hline C & 1 & 6 & 6 & 0 & 3 \\
\hline D & 4 & 3 & 0 & & 3 \\
\hline $\mathbf{E}$ & 4 & 0 & 2 & 4 & \\
\hline
\end{tabular}

Here we see that all zeros are either assigned or crossed out. That is, the total assigned zero's is 5 which is equal to the number of rows or columns. And the solution is $(1,5),(2,1),(3,4),(4,3),(5,2)$.

And the total cost $=1+0+2+1+5=09$.

\section{Solve the following assignment problem using Matrix One's Assignment Method.}

Consider the problem of assigning five jobs to five persons. The assignment costs are given below.

\begin{tabular}{|l|lllll|}
\hline \multirow{2}{*}{ Persons } & \multicolumn{5}{|c|}{ Jobs } \\
& 1 & 2 & 3 & 4 & 5 \\
\hline A & 8 & 4 & 2 & 6 & 1 \\
B & 0 & 9 & 5 & 5 & 4 \\
C & 3 & 8 & 9 & 2 & 6 \\
E & 4 & 3 & 1 & 0 & 3 \\
\hline
\end{tabular}

Determine the optimum assignment schedule and minimum assignment cost.

\section{Solution:}

Step 1: Find the minimum cost of each row and then divide each element of each row of the matrix by its minimum cost.

\begin{tabular}{llllll|}
\multicolumn{1}{c}{} & 1 & 2 & 3 & 4 & 5 \\
\cline { 2 - 6 } B & 8 & 4 & 2 & 6 & 1 \\
B & 0 & 0 & 0 & 0 & 0 \\
D & 1.5 & 4 & 4.5 & 1 & 3 \\
E & 0 & 0 & 0 & 0 & 0 \\
& 1.8 & 1 & 1.6 & 1.8 & 1 \\
\hline
\end{tabular}

Step 2: Find the minimum cost of each column and then divide each element of the column by its minimum cost.

\begin{tabular}{l|lllll}
\multicolumn{1}{c}{} & 1 & 2 & 3 & 4 & 5 \\
\cline { 2 - 5 } B & 0 & 0 & 0 & 0 & 0 \\
C & 0 & 0 & 0 & 0 & 0 \\
D & 0 & 0 & 0 & 0 & 0 \\
E & 0 & 0 & 0 & 0 & 0 \\
\hline
\end{tabular}

Here we see that there is no one's term to assign in the above matrix. So we can't solve this problem by MOAmethod. 
New Proposed Method for Solving Assignment Problem and Comparative Study with the Existing...

Here new proposed method is introduced for solving assignment problem. Also an example using the proposed method and two existing methods is examined and the optimal solutions are compared among three methods and the optimal solutions by three methods are same result.

Table 1.2: Comparison of Optimal Values of three Methods

\begin{tabular}{|l|l|l|l|l|}
\hline Example & HA-Method & MOA-Method & Proposed Method & Optimum \\
\hline 01 & 09 & - & 09 & 09 \\
\hline
\end{tabular}

Therefore we conclude that this new Proposed Method is effective for solving assignment problem.

\section{Conclusion}

In this paper, we presented a new method for solving Assignment problem. Initially, we explained the proposed algorithm and showed the efficiency of it by numerical example. And we get the optimal solution which is same as the optimal solutions of HA-method and MOA-method. Therefore this paper introduces a different approach which is easy to solve Assignment problem.

\section{References}

[1]. D.F. Votaw, 1952, A. Orden, The perssonel assignment problem, Symposium on LinearInequalities and Programming, SCOOP 10, US Air Force, pp. 155-163.

[2]. H.W. Kuhn, 1955, The Hungarian method for the assignment problem, Naval Research Logistics Quarterly 2 (1\&2) 83-97 (original publication).

[3]. M.S. Bazarra, John J. Jarvis, Hanif D. Sherali, 2005, Linear programming and networkflows

[4]. B.S. Goel, S.K. Mittal, 1982, Operations Research, Fifty Ed., 2405-2416.

[5]. Hamdy A. Tsaha, 2007, Operations Research, an introduction, $8^{\text {th }}$ Ed..

HadiBasirzadeh 2012, Applied Mathematical Sciences, 6(47), 2345-2355.

Singh S., Dubey G.C., Shrivastava R. (2012) IOSR Journal of Engineering, 2(8), 01-15.

Turkensteen M., Ghosh D., Boris Goldengorin, Gerard Sierksma, 2008 European Journal of Operational Research, 189, 775-788

New Proposed Method for Solving Assignment Problem and Comparative Study with the Existing Methods

Zimmermman H.J. (1996) Fuzzy Set Theory and its Applications, $3^{\text {rd }}$ ed., KluwerAcademic, Boston.

Pentico D.W. (2007) European Journal of Operation Research, 176, 774-793.

Singh S. (2011) International Journal of Operation Research and Information System,

3(3), 87-97.
AnshumanSahu, RudrajitTapador, 2007, Solving the assignment problem using Genetic Algorithm And Simulated annealing, IJAM.

[15]. Shayle R. Searle, 2006, Matrix algebra useful for statistics, John Willey. 